\title{
El español en el paisaje lingüístico del Reino Unido
}

\section{The Linguistic Landscape in Spanish in the UK}

\author{
RAQUEL NAVAS \\ UNIVERSITY OF WARWICK \\ r.navas@warwick.ac.uk \\ https://orcid.org/0000-0002-1129-4257
}

\begin{abstract}
Resumen
El objetivo principal de este artículo es explorar el papel del paisaje lingüístico $(\mathrm{PL})$ como herramienta de conexión con la lengua y las culturas hispánicas en un proyecto con estudiantes de español de una institución de educación superior británica. Con el fin de solventar la falta de vínculo entre las asignaturas de lengua y las de cultura, estas últimas impartidas en inglés, se examina cómo integrar la reflexión lingüística y léxico-gramatical en una asignatura sobre identidad textual mediante el uso de cartelería, señales y otras muestras de español en la comunidad donde viven y estudian los estudiantes.
\end{abstract}

Palabras clave: paisaje lingüístico, aprendizaje fortuito, aprendizaje fuera del aula, adquisición de segundas lenguas.

\begin{abstract}
The objective of this article is to explore the role of the Linguistic Landscape (LL) as a tool to connect Spanish language with its culture(s) through a project with undergraduate students at a British university. The purpose of the project is to bridge the gap between language modules and culture modules by integrating reflections about linguistic, lexical, and grammatical items on a culture module about textual identity, using signage and other environmental print samples in Spanish in the community where students live and study. Keywords: Linguistic Landscape, incidental learning, out-of-class learning, Second Language Acquisition.
\end{abstract}




\section{Introducción}

A menudo, en los departamentos de lenguas extranjeras de las universidades británicas se produce una distinción entre asignaturas de lengua y asignaturas de cultura. Las primeras son aquellas en las que se estudia la lengua a través de la traducción, la gramática y las comprensiones y expresiones orales-aurales y lectoras-escritas. Las segundas son aquellas en las que se estudia cualquier ítem de historia, cultura, literatura, cine u otros, del mundo hispano. Estas últimas se imparten en L1 y se evalúan en dicha lengua, normalmente a través de ensayos en los que, aunque algunas de las lecturas están disponibles en español, la LE no se advierte ni en el proceso de investigación ni en el de redacción.

Esta división entre los dos tipos de asignatura, que es visible y aceptada por el profesorado y el alumnado, provoca que estos últimos no conecten los contenidos de los dos tipos de asignaturas, sino que permanecen en compartimentos estancos. De ahí surgió la pregunta ¿cómo crear puentes entre los dos tipos de asignatura? ¿cómo solventar la falta de conexión entre lengua y cultura? Es decir, ¿cómo conseguir reflejar el mundo real que está más allá del aula, en el que la lengua y las culturas forman un todo?

En este artículo se presentará el concepto de paisaje lingüístico (PL, en adelante) y algunos estudios relevantes sobre este y la concienciación lingüística, la adquisición de lenguas extranjeras ( $A S L$, en adelante) y la enseñanza del español. Posteriormente, se presentará un proyecto de PL llevado a cabo con estudiantes de grado de la Universidad de Warwick y se mostrarán ejemplos de reflexiones lingüísticas dentro y fuera del aula.

\section{El paisaje lingüístico: definición y una selección de trabajos}

En la literatura sobre PL se considera el trabajo de Landry y Bourhis (1997) como el primero en identificar el término. Los autores lo definen como la visibilidad de las lenguas en espacios públicos y comerciales, es decir, cualquier tipo de escritura que vemos en espacios públicos: señales en la carretera, vallas publicitarias, nombres de calle, rótulos de establecimientos comerciales y edificios gubernamentales. No obstante, se encuentran definiciones más actualizadas en Cenoz y Gorter (2008: 257), "the Linguistic Landscape refers to all language items that are visible in a specified part of the public space" y Ben-Rafael (2009: 40), "the notion of Linguistic Landscape refers to linguistic objects that mark the public spaces, outside private homes" ya que estas engloban muestras multimedia o en movimiento, propias de los avances tecnológicos de los últimos años. El PL es un campo que vive a caballo entre la sociología, la sociolingüística, la psicología social, la geografía y las ciencias de la información y la comunicación (Sebba, 2010) y es de reciente creación. 
En los últimos 15 años han aparecido numerosos estudios que usan el PL en el aula como herramienta educativa para fomentar la concienciación y la sensibilidad multilingüística. El trabajo de Landry y Bourhis (1997) ya mencionado, como se ha indicado, se propone observar la percepción del alumnado canadiense en cuanto a la presencia de la lengua francesa en su comunidad, Quebec. Dagenais et al., (2009); Clemente, Andrade y Martins (2012) pretenden concienciar a estudiantes de educación primaria sobre diversidad lingüística; Hancock (2012) examina las percepciones sobre el PL de Edimburgo que poseen estudiantes de educación con el objetivo de promover el pensamiento crítico sobre creencias, valores y estereotipos; Hewitt-Bradshaw (2014) explora el uso del PL como recurso educativo en clases de alfabetización y Malinowski (2016) diseña un curso multidisciplinar que relaciona el contexto académico, el aula, y el espacio real de fuera de esta. Además, a partir de 2020 empiezan a surgir los primeros monográficos sobre PL y educación (Niedt y Seals, 2020; Malinowski, Maxim y Dubreil, 2020 y Krompák, Fernández-Mallat y Meyer, 2021).

Existen, además, varios estudios que usan los mensajes del entorno como herramienta para la ASL. En el estudio teórico de Cenoz y Gorter (2008), los autores examinan el uso potencial del PL como fuente de input en LE. En cuanto a estudios prácticos, Sayer (2010) describe un proyecto pedagógico en México, en el que se examina y se debate la presencia del inglés en la ciudad de Oaxaca y se presenta una taxonomía de los usos de la lengua en esta comunidad. El trabajo de Malinowski (2010) provee actividades e ideas para trabajar el PL en la enseñanza de LE. El autor plantea una yuxtaposición en 3; es decir, dividir las secuencias didácticas a en función de las diferentes maneras en las que se puede estar en el mundo, interactuar con este y conocerlo. El estudio de Rowland (2013) debate los beneficios pedagógicos de un proyecto de PL del inglés con estudiantes de grado japoneses, con el fin de aportar materiales a otros profesores de inglés y el de Chesnut, Lee y Schulte (2013) describe las percepciones que experimentan estudiantes coreanos con el objetivo de reflexionar sobre lo que se ha aprendido y sobre las dificultades con las que se han encontrado. Barrs (2016) realiza una exploración del inglés en el PL de Japón intentando dar respuesta a cuestiones tales como ¿cuánto inglés se usa de forma cotidiana en Japón? o ¿qué procesos se llevan a cabo a la hora de nombrar un producto japonés?

En cuanto al PL y el español como LE se ha producido un auge de proyectos en los últimos diez años. En primer lugar, destaca el trabajo de Esteba Ramos (2013), cuyo objetivo es el de reflexionar sobre los beneficios del uso del PL aplicado a la enseñanza del español como LE y, además, proponer algunas prácticas específicas. Seguidamente, LlopisGarcía (2015) y sus compañeros de la Universidad de Columbia desarrollan el proyecto Twitter and The City: El paisaje lingüístico de ÑYC con el objetivo de fomentar el aprendizaje más allá del aula, simular un contexto de inmersión del español en la ciudad de Nueva York e interactuar y 
fomentar el aprendizaje crítico y autónomo. El proyecto sobre el español en la capital inglesa, En un lugar de Loñdres, de Lourdes Hernández Martín (Hernández- Martín y Skandries, 2020) tiene como objetivo promover que el estudiantado salga del aula y examine el uso de la lengua dentro del contexto multilingüe en que se encuentran los espacios públicos de esta ciudad. Todo ello con el fin de fomentar que los estudiantes busquen el impacto del español y, además, de que estos registren el español como una lengua que pertenece ya a la ciudad y que es hablada por los habitantes de Londres. Aladjem y Jou (2016) presentan un proyecto de PL de estudiantes de grado a través de Facebook con el objetivo de complementar el aprendizaje de la lengua que estos reciben en el aula y de animarlos a continuar con el estudio de esta. Ma (2018) presenta varias actividades didácticas de niveles diferentes y debate cómo el PL puede fortalecer tanto el estudio de la lengua como el aprendizaje fuera del aula. Y Bruzos (2020) describe un proyecto en el que estudiantes de español de grado exploran el PL de Madrid cuyo objetivo es romper con estereotipos culturales asociados a la idea de España, gracias a la posición de los estudiantes como investigadores que interactúan con la realidad política y social de la ciudad.

\section{El proyecto}

Este es un proyecto anual que se inició en el curso 2016-2017 con estudiantes de primer año del grado de Lenguas Modernas de la Universidad de Warwick, miembro de la liga de universidades de élite Russell Group. Dichos aprendientes estudian diferentes combinaciones de lenguas, hasta un máximo de tres, todas ellas con español. El alumnado de este grupo cursa 4 horas a la semana de lengua en la asignatura Español avanzado, que corresponde aproximadamente a un B2 según los niveles del MCER (Consejo de Europa, 2002), o 5 horas a la semana de Español para principiantes, que corresponde a los niveles A1 (inicio del curso) -B1 (final del curso). El grupo cursa, además, una asignatura de cultura, Lengua, textos e identidad de 2 horas a la semana, impartida en inglés y en la que se presenta una introducción a la sociolingüística del español. Es en esta asignatura donde se enmarca este proyecto.

Durante varias semanas se realiza una primera introducción a conceptos como lengua, acento y dialecto/variedad, cambio de código, préstamos lingüísticos, y bilingüismo y diglosia. Además, se tratan de manera introductoria las variedades del español peninsular y del español de América, otras lenguas en contacto con este, el spanglish y el PL en español del Reino Unido.

Durante las primeras semanas de clase se expusieron los objetivos del proyecto, estudiar cuándo, dónde, cómo y por qué aparece el español en el PL del Reino Unido y se propuso a los estudiantes que tomaran fotografías de cualquier aducto en esta lengua que encontraran en su día a día. Se pidió que publicaran estas muestras en Twitter atendiendo a las 
preguntas ¿qué es?, ¿dónde lo has encontrado?, ¿por qué te ha llamado la atención? ¿por qué usa el español? y ¿qué has aprendido?

Posteriormente, se proporcionó una base teórica sobre PL y se eligieron aquellas fotografías y tuits que podían aportar discusión. En el aula se debatieron tanto los usos sociales, significado y categorización del español en el área como aspectos léxico-gramaticales y sociolingüísticos.

\section{Reflexiones}

Usando una taxonomía inicial que atendía a aspectos como la disposición de lenguas, lugar encontrado, función del mensaje, tamaño y color de fuente, entre otras, se pudieron debatir cuestiones léxicas, gramaticales, sociolingüísticas y culturales, cuyos ejemplos veremos a continuación.

En la Fotografía 1 se observa que la palabra tapas aparece en el menú de una pizarra de un restaurante asiático cercano al campus. En ella vemos que la intención de los autores de la muestra es describir el término Dim sum (multitud de pequeños platos) para aquellas personas que desconocen su significado. Esta fotografía llevó a la reflexión y despertó el interés del estudiantado, que descubrió que tapas estaba empezando a usarse como préstamo lingüístico del español al inglés con el significado de pequeñas porciones de comida de cualquier tipo. Para corroborar esta teoría, se pidió a los estudiantes que estuvieran atentos al uso de tapas en su PL y descubrimos dos nuevos ejemplos; uno de comida india (Fotografía 2) y uno de comida británica (Fotografía 3).

Fotografía 1: Dim sum (tapas). @usuario3 (2016)

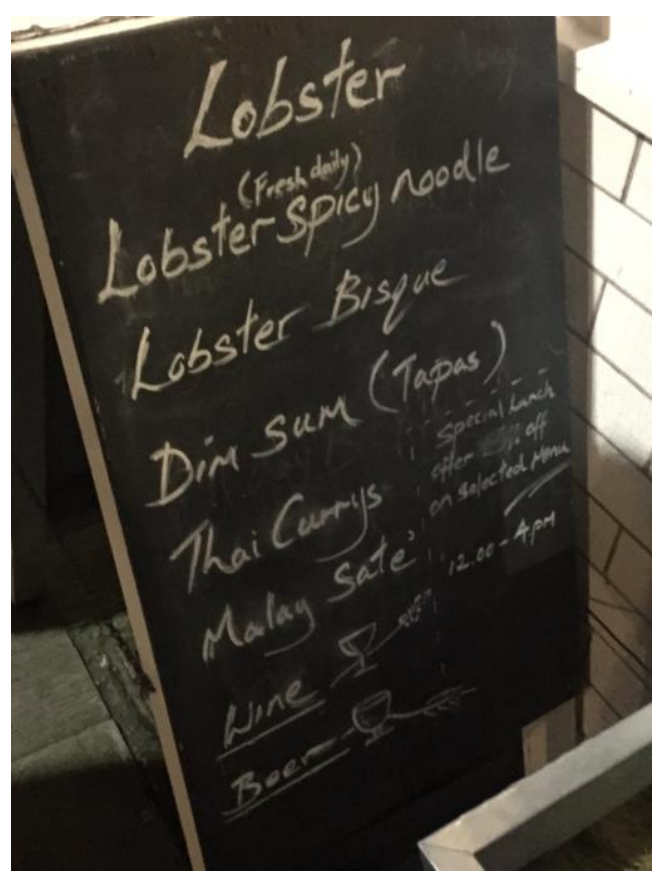


Fotografía 2: Indian Tapas. Propiedad de la autora (2019)

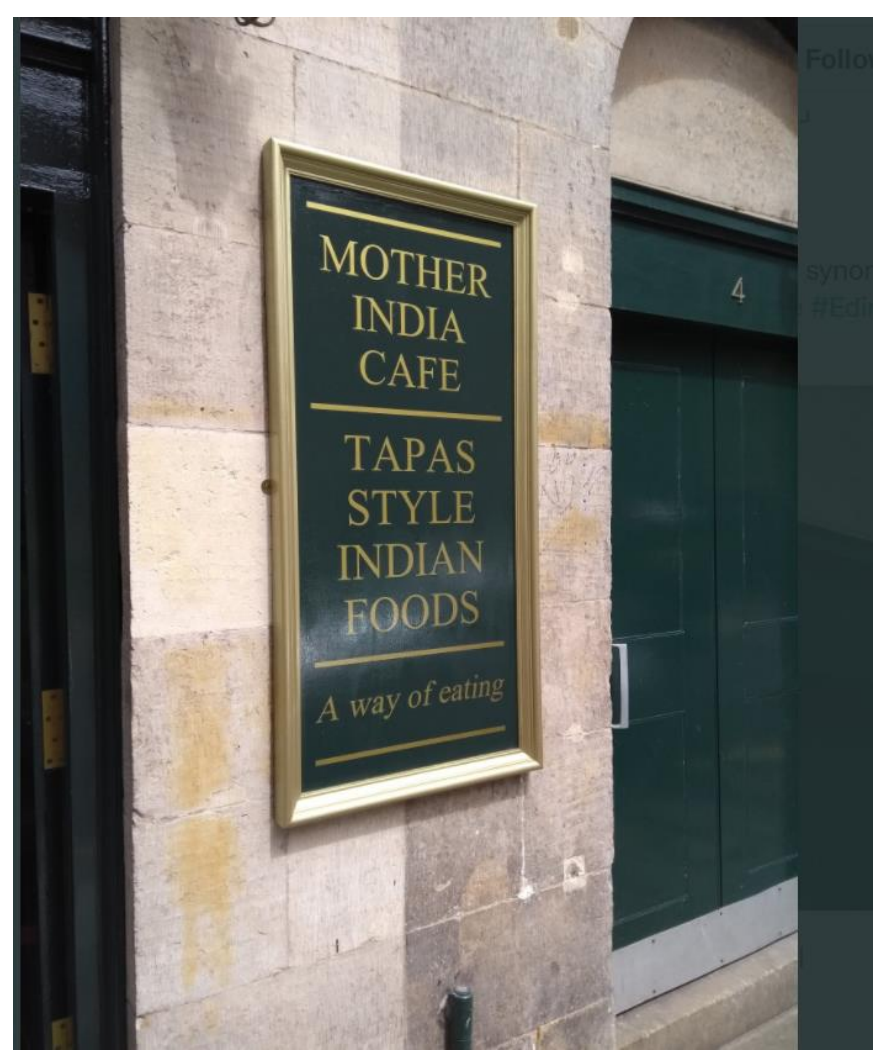

Fotografía 3: Red Lion (tapas). @usuario20 (2019)

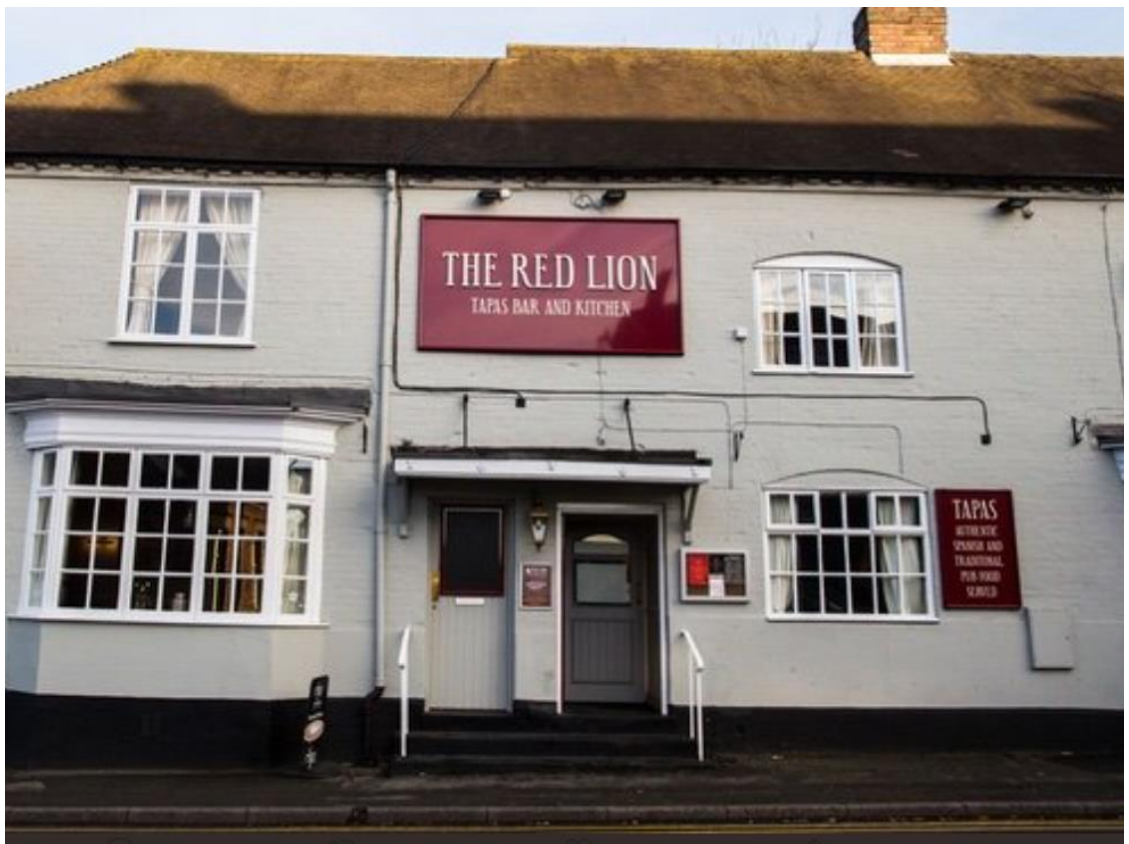


La fotografía 1 promovió aprendizaje autónomo en los estudiantes ya que fueron ellos mismos quienes encontraron las muestras y quienes llegaron a la conclusión de la posibilidad de un nuevo préstamo lingüístico del español al inglés. Por lo tanto, se trabajaron competencias y habilidades investigadoras y se usaron muestras de lenguas reales. Estas características se verán repetidas en los ejemplos siguientes.

En la fotografía 4, que muestra un escaparate de la tienda Mango en un centro comercial de la ciudad de Birmingham, se aprecia que el mensaje en catalán es perceptiblemente más visible ya que aparece resaltado en negrita y dispone de un tamaño de fuente superior al resto de lenguas. Esto llevó al aula discusiones sobre disposición de textos en el PL. Scollon y Wong Scollon (2003) afirman que la disposición de la lengua en el mensaje indica el protagonismo de esta: "When there are two languages in use in bilingual notices, the code preference system positions the uppermost or left-most of two languages as the preferred code, the lower or right-most language as peripheralized" (p.158). Se ejemplificó con la "Ley 101" de Quebec, en Canadá, que requiere que el francés aparezca de forma predominante en la cartelería y señalización, por encima del inglés (Malinowski, 2009).

Esto nos llevó a reflexionar sobre identidad de marca y se llegó a la hipótesis de que la compañía Mango busca identificarse como marca catalana, o quizás, como marca de Barcelona, debido a la concepción que se tiene de esa ciudad como sinónimo de cosmopolitismo en el Reino Unido.

Fotografía 4: Escaparate de Mango. Propiedad de la autora

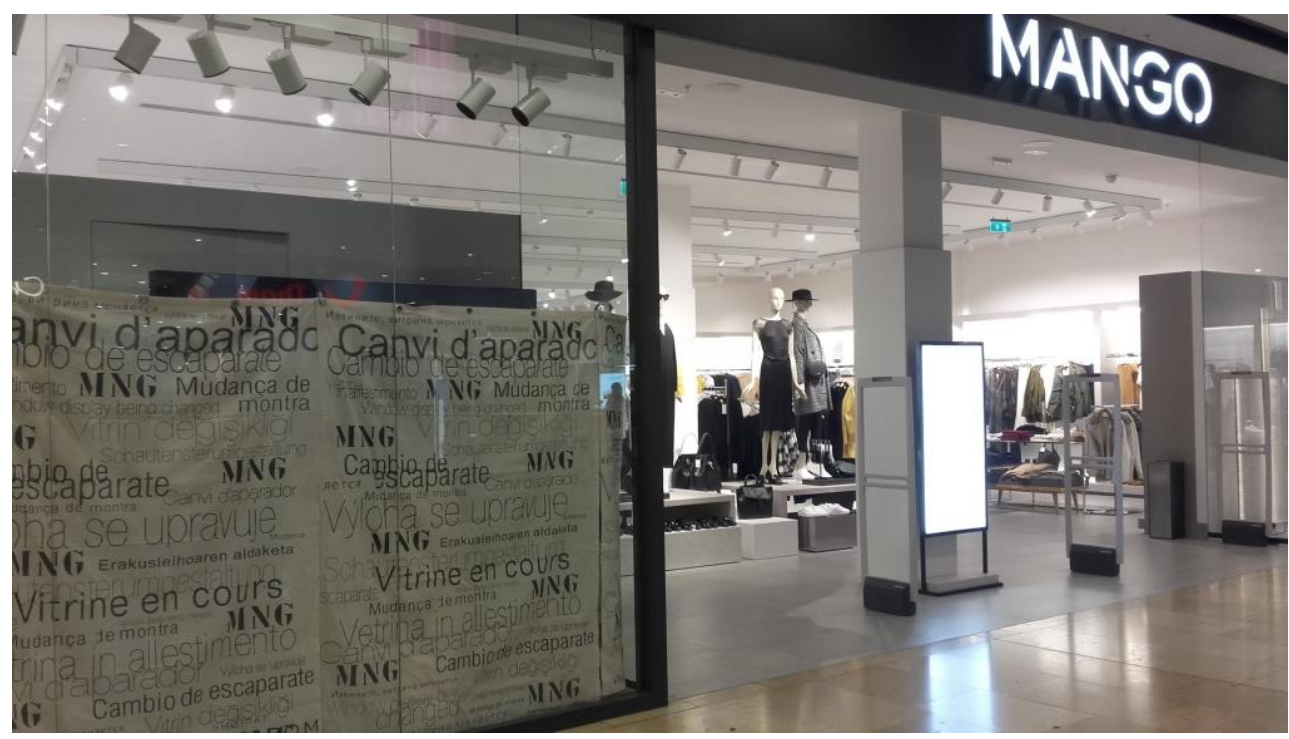

En la Fotografía 5 se observa que nuestra estudiante define dulce de leche como un préstamo lingüístico ya que se ha usado el nombre del producto en español y se ha añadido una pequeña explicación en inglés (velvety vanilla caramel spread: crema de vainilla y caramelo para untar). Sin 
embargo, lo que llamó la atención de la estudiante y lo que llevamos al aula fue la discusión sobre estereotipos y representaciones de las culturas hispanas. Como se observa, el supermercado lo describe como lo mejor de España, siendo este un producto mundialmente conocido como argentino. Esto condujo a un nuevo estadio, la interacción con el $\mathrm{PL}$, ya que la estudiante escribió al supermercado con información sobre el origen y el producto en cuestión para que este rectificara el error.

Fotografía 5: Dulce de leche. @usuario10 (2017)

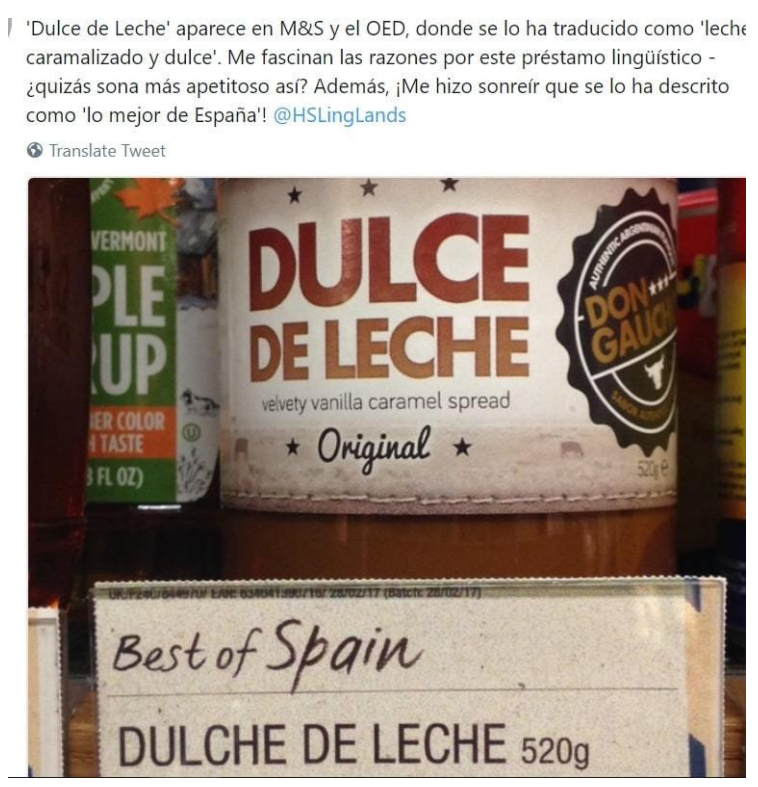

La Fotografía número 6 muestra un cartel informativo en la estación central de la ciudad de Birmingham, cercana al campus. En ella se observa un mensaje que recuerda a los viajeros que no olviden ningún objeto en el tren. Este mensaje contiene una traducción duplicada (Reh, 2004), es decir que la información que se proporciona es la misma en las dos lenguas.

Esta fotografía que, en principio, no parece aportar información relevante, suministró una gran cantidad de debate en el aula:

(1) Los estudiantes actuaron como correctores y observaron que el mensaje contenía algunas erratas como el símbolo de interrogación inicial o el uso de boletas en femenino.

(2) Se debatieron las diferentes variedades del español. Por proximidad geográfica con España se suelen encontrar muestras de esta variedad, sin embargo, el uso de boletas (boletos) indica que podría ser una variedad latinoamericana. Posteriormente, se reflexionó que el uso de tú y del término dinero podría indicar que se trataba de una variedad peninsular.

(3) Esto nos llevó al tercer estadio en el que se pidió a los estudiantes que se fijaran atentamente en las marcas de color verde en la fotografía. El estudiante que tomó la 
fotografía no era consciente de que aparecen tachadas tanto la $s$ final de has como la $a$ de boletas en rotulador, lo que nos llevó a asumir que un o unos hablantes nativos no estaban conformes con el uso de tú ni con el uso de boletas en femenino. Este es un ejemplo claro de inconformismo lingüístico; el vandalismo en señales del PL demuestra que los habitantes de la región no están conformes con el uso de la lengua y han llevado en muchas ocasiones a un cambio lingüístico en la región. Gracias a esta fotografía, se trabajó el concepto de políticas lingüísticas y se ejemplificó con los casos de Bélgica, donde el vandalismo del PL (Landry y Bourhis, 1997) ayudó a la creación de zonas lingüísticas diferenciadas y el de Cataluña, cuya cartelería está en constante batalla entre un uso bilingüe español-catalán, monolingüe español o monolingüe catalán.

Fotografía 6: ¿Has olvidado algo? @usuario 11 (2017)

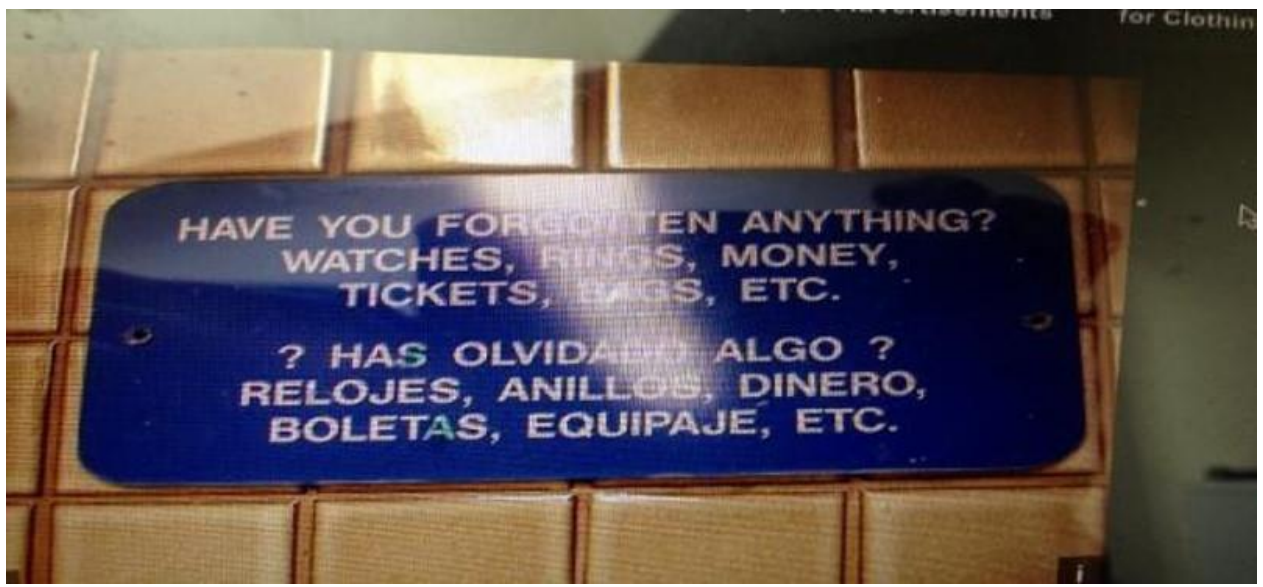

Otras reflexiones léxico-gramaticales que se produjeron en el aula estuvieron relacionadas con el aprendizaje de nuevo vocabulario, como la palabra pastor en una taquería de Londres, (Fotografía 7) y el sufijo -ita como marca de objeto de pequeño tamaño de las cervezas Coronita, (Fotografía 8). 
Fotografía 7: Tacos El pastor. @usuario5 (2016).

@ HSLingLands Un restaurante en Borough Market cerca de London Bridge. Aprendí la palabra 'pastor' pero ¡dudo que haya 'pastores' en Londres!

(6) Translate Tweet

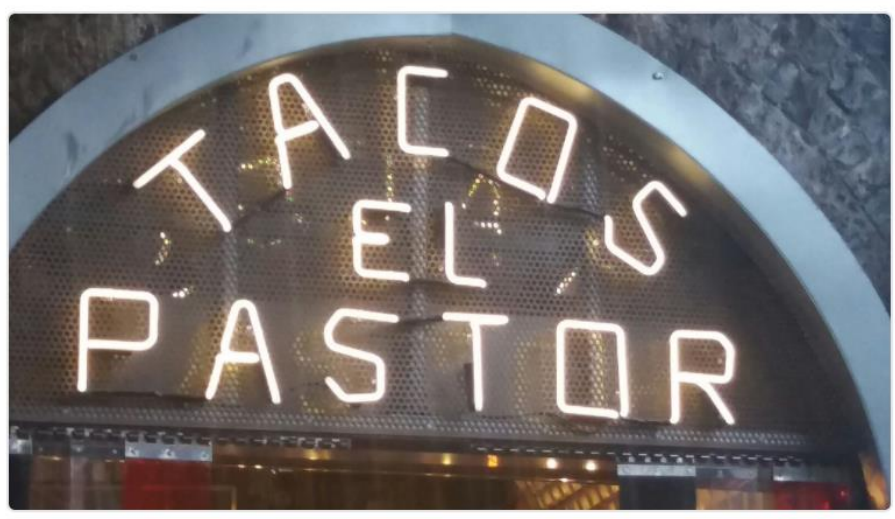

๑ $1 \begin{array}{llll}1 & \bullet & & \end{array}$

Fotografía 8: Coronita (sufijo -ita). @usuario3 (2016 b)

la diferencia en la tamaña de los productos. Eso es algo muy español y me intrigaba mucho cuando lo vi

(6) Translate from Spanish

Q 1 1

You Retweeted

@ HSLingLands Vi botellas pequeñas de Corona en Aldi. Creía que era muy eficaz que Corona pusiera 'ita' al fin de su nombre para expresar

(6) Translate from Spanish

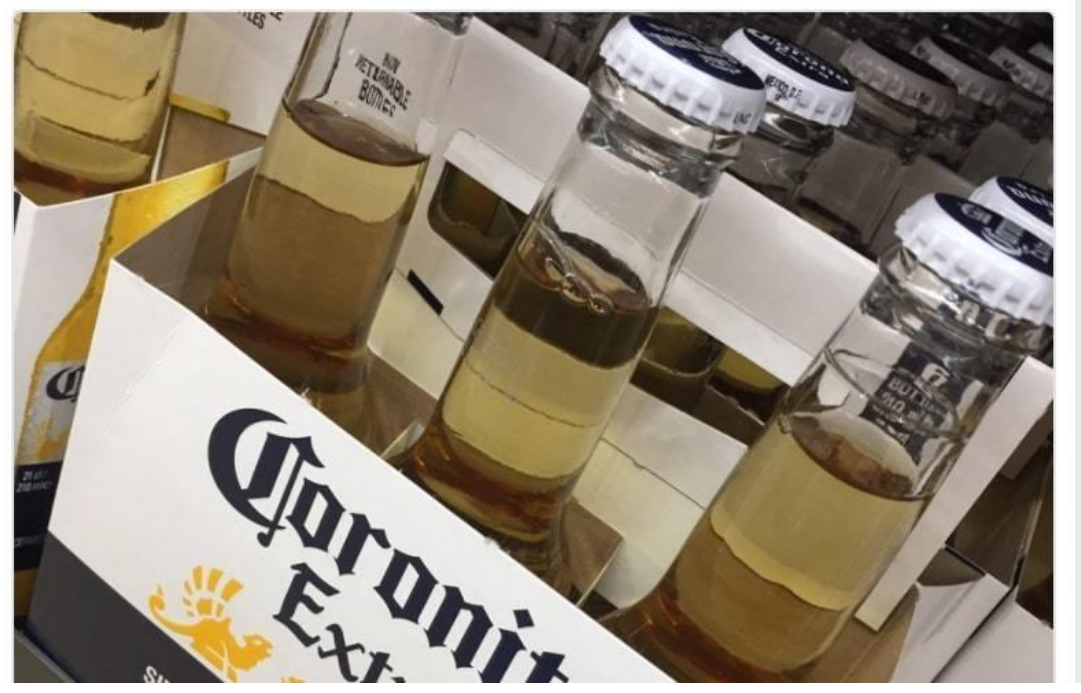




\section{Conclusiones}

En este artículo hemos resumido algunas características y beneficios del análisis del PL en el estudio del español como LE, ya que este ha demostrado ser una herramienta que proporciona infinitas posibilidades de estudio de la lengua y las culturas hispanas:

(1) Se ha trabajado información sociolingüística. Se han analizado y ejemplificado términos como préstamos léxicos, cambio de código, variedades del español, políticas lingüísticas, lengua e identidad, entre otros.

(2) Se han trabajado conceptos léxico-gramaticales. Se ha reflexionado sobre el uso de diminutivos, de complementos indirectos, participios, etcétera.

(3) Se ha colaborado a despertar concienciación sobre las culturas hispanas y las diferentes variedades del español.

Además, se ha promovido el pensamiento crítico, el aprendizaje autónomo y competencias y habilidades investigadoras ya que son los propios estudiantes quienes consiguen las muestras. También ayuda a construir la cultura de la lengua extranjera y, lo que es más importante, los estudiantes han trabajado y han estado en contacto constante con muestras auténticas de lengua, a pesar de cursar una asignatura impartida en inglés y de encontrarse en un contexto de no inmersión. Cenoz y Gorter (2008) afirman que el PL proporciona visibilidad a las lenguas, esto favorece la motivación en el estudiante, ya que siente que el español es parte de su contexto, de su día a día y de su vida y que la lengua que estudia no es un concepto suelto que mide su capacidad de estudio, sino que está interconectada con la cultura y con la realidad, con las que puede jugar e interactuar (Navas, 2021).

Asimismo, el PL proporciona aprendizaje incidental en el estudiante ya que este no se espera aprender de él, este promueve que "la enseñanza y el aprendizaje se extiendan por cualquier rincón de la ciudad y que los alumnos se den cuenta de que su entorno vital también es un aula de español" (Ma, 2018) Igualmente, el estudio del PL aporta una gran flexibilidad tanto en el aula como fuera de ella ya que en cada proyecto y en cada curso las fotografías que se toman son diferentes y muestran partes sin explorar del uso del español en el Reino Unido.

Por ello se propone desde aquí la creación de más proyectos que exploten el uso de PL como herramienta pedagógica y que nos lleven a descubrir nuevos tesoros lingüísticos escondidos. 


\section{BiBLIOGRAFÍA}

Aladjem, R. y Jou, B. (2016): "The linguistic landscape as a learning space for contextual language learning". Journal of Learning Space, 5 (2), pp.66-70.

BARRS, K. (2016): "The typology of English in Japanese society: Learning from the Linguistic Landscape". Electronic Journal of Foreign Language Teaching, 17(1), pp.7-15.

Ben-Rafael, E. (2009): "A Sociological Approach to the Study of Linguistic Landscapes". E. Shohamy y D. Gorter (eds.), Linguistic Landscape: Expanding the Scenery. Nueva York y Londres: Routledge, pp. 40-54.

Bruzos, A. (2020): "Linguistic Landscape as an Antidote to the Commodification of Study Abroad Language Programs: A Case Study in the Center of Madrid". D. Malinowski, H. Maxim y S. Dubreil (eds.), Language Teaching in the Linguistic Landscape: Mobilizing Pedagogy in Public Space, Springer, pp. 253-293.

Cenoz, J. y Gorter, D. (2008): "The Linguistic Landscape as an additional source of input in second language acquisition". IRAL- International Review of Applied Linguistics in Language Teaching, 46, pp. 267287.

Chesnut, M.; LeE, V. y SChulte, J, (2013): "The language lessons around us: Undergraduate English pedagogy and Linguistic Landscape research". English Teaching: Practice and Critique, 12 (2), pp. 102120.

Clemente, M.; Andrade, A. I. y Martins, F. (2012): "Learning to read the world, learning to look at the Linguistic Landscape: a primary school study". C. Hélot, M. BARNI, R. JANSSENS y C. Bagna (eds.), Linguistic Landscapes, multilingualism and social change, Frankfurt: Peter Lang, pp. 267-285.

Dagenais, D.; Moore, D.; Sabatier, C., Lamarre, P. y Armand, F. (2009): "Linguistic Landscape and language awareness". E. SHOHAMY y D. GORTEr (eds.), Linguistic Landscape: Expanding the Scenery. Nueva York y Londres: Routledge, pp.253-269.

Esteba Ramos, D. (2013): “¿Qué te dice esta ciudad? Modelos de reflexión y propuestas de actuación en torno al paisaje lingüístico en la clase de Español Lengua Extranjera". L. Ruiz Miyares (ed.), Actualizaciones en Comunicación Social. Santiago de Cuba: Ediciones del Centro de Lingüística Aplicada, pp.474-479.

HANCOCK, A. (2012): "Capturing the Linguistic Landscape of Edinburgh: a pedagogical tool to investigate student teachers' understandings of cultural and linguistic diversity". C. Hélot, M. Barni, R. Janssens y C. Bagna (eds.) Linguistic Landscapes, multilingualism and social change. Frankfurt:Peter Lang, pp.249-266.

Hernández Martin, L. y SKANDRIES, P. (2020): "Taking the Foreign out of Language Teaching: Opening up the Classroom to the Multilingual City". D. Malinowski, H. Maxim, S. Dubreil (eds.), Language 
Teaching in the Linguistic Landscape: Mobilizing Pedagogy in Public Space. Springer, pp. 253-293.

HeWitT-BRAdSHAW, I. (2014): "Linguistic Landscape as a language learning and literacy resource in Caribbean creole contexts". Caribbean Curriculum, 22, pp. 157-173.

Krompák, E.; Fernández-Mallat, V. y Meyer, S. (eds.) (2021): Linguistic Landscape and Educational Spaces. Bristol, Multilingual Matters.

LANDRY, R. y BOURHIS, R. (1997): "Linguistic Landscape and Ethnolinguistic Vitality", Journal of Language and Social Psychology, 16 (1), pp.2349.

LLOPIS-GARCÍA, R. (2015): ÑYC as a Canvas for Spanish Language, Culture, and Identity. [vídeo]. Columbia Language Resource Center. https://www.youtube.com/watch?v=3X81KCvKYdA\&feature=youtu. $\underline{\text { be }}$

MA, Y. (2018): "El paisaje lingüístico: una nueva herramienta para la enseñanza de E/LE". Foro de profesores de E/LE, 14, pp. 153-163.

MALINOWSKI, D. (2010): "Showing seeing in the Korean linguistic cityscape". E. Shohamy, E. Ben-RAfael y M. BARni (eds.), Linguistic Landscape in the city. Bristol: Multilingual Matters, pp.199-215.

MALINOWSKI, D. (2016): "Localizing the transdisciplinary in practice: A teaching account of a prototype undergraduate seminar on Linguistic Landscape". L2 Journal, 8, pp. 100-117.

MALINOWSKI, D.; MAXIM, H.; DUBREIL, S. (eds.) (2020): Language Teaching in the Linguistic Landscape: Mobilizing Pedagogy in Public Space. Springer.

NAVAS, R. (2021): El paisaje lingüístico: vivir el español fuera del aula. Colección monografías de ASELE, 11. ASELE.

Niedt, G. y SeAls, C. (eds.) (2020): Linguistic Landscapes Beyond the Language Classroom. London: Bloomsbury.

RoWLAND, L. (2013): "The pedagogical benefits of a Linguistic Landscape project in Japan". International Journal of Bilingual Education and Bilingualism, 16 (4), pp .494-505.

SAYER, P. (2010): "Using the Linguistic Landscape as a pedagogical resource". ELT Journal 64 (2), pp. 143-154.

SCollon, R. y Wong SColLon, S. (2003): Discourses in Place. Language in the Material World. Londres, Routledge

SebBA, M. (2010): "Linguistic Landscapes: A Comparative Study of Urban Multilingualism in Tokyo by Peter Backhaus". Writing Systems Research, 2 (1), pp. 73-76. 